\title{
Duration and Clinical Relevance of Postantibiotic Effect in Relation to the Dosing Interval
}

\author{
JAN G. DEN HOLLANDER, ${ }^{1 *}$ KURT FUURSTED,${ }^{2}$ HENRI A. VERBRUGH,${ }^{1}$ AND JOHAN W. MOUTON ${ }^{1}$ \\ Department of Medical Microbiology and Infectious Diseases, University Hospital Rotterdam, Dijkzigt, Rotterdam, \\ The Netherlands, ${ }^{1}$ and Department of Clinical Microbiology, Statens Serum Institut, Copenhagen, Denmark ${ }^{2}$
}

Received 28 January 1997/Returned for modification 27 July 1997/Accepted 23 December 1997

\begin{abstract}
The influence of half-life on the postantibiotic effect (PAE) of tobramycin against Pseudomonas aeruginosa and Staphylococcus aureus was investigated during one dosing interval. Tobramycin half-lives of 0.5 to $2.5 \mathrm{~h}$ were simulated in an in vitro model, and the PAE was determined by an enzymatic inactivation method at different time points, i.e., when the tobramycin concentrations were $20 \times, 5 \times$, and $1 \times$ the MIC. At the time point during therapy when the tobramycin concentrations had declined to $1 \times$ the MIC, at a tobramycin half-life of $0.5 \mathrm{~h}$, the times of the PAEs were approximately 0.7 and $1.7 \mathrm{~h}$ for $P$. aeruginosa and $S$. aureus, respectively, and the PAE disappeared completely at half-lives corresponding to those found in humans (i.e., 2 to $2.5 \mathrm{~h}$ ). The PAE itself cannot be fully explained by the presence of free intrabacterial tobramycin or the emergence of resistant subpopulations. The explanation for the disappearance of the PAE during the dosing interval may therefore be explained by the repair of sublethal damage. Since the standard method of determining the PAE in animal models is somewhat different from the method used for measurement of the PAE in vitro, the conditions under which the PAE is measured in vivo were also simulated in the in vitro model. This resulted in PAEs longer than those found by the standard method of obtaining in vitro PAE measurements. We conclude that the PAE of tobramycin, as determined by conventional in vitro methods, has virtually no clinical importance. PAEs determined in vivo may have some clinical relevance, but they are probably primarily caused by sub-MIC effects.
\end{abstract}

The clinical relevance of the postantibiotic effect (PAE) determined in vitro remains questionable. The main problem is that the PAE is measured as delayed bacterial growth after a short on-off exposure to an antibiotic for 1 or $2 \mathrm{~h}$ (4). Such exposure does not reflect the situation in humans under clinical conditions, where bacteria are exposed to antibiotic concentrations that decline only slowly over time, with half-lives of up to several hours. Nevertheless, the PAE has been used to support the lengthening of the dosing interval for aminoglycosides $(14,15,20,22)$, since it is assumed that the PAE would significantly delay bacterial regrowth after the antibiotic concentration falls below the MIC (2).

Recently, we showed that the PAE completely disappeared during one interval of dosing with tobramycin simulated in an in vitro pharmacokinetic model (5). The PAE determined at the peak concentration $(20 \times$ the MIC) correlated well with the PAE measured under standard conditions, but it declined to zero at the end of a dosing interval. There are several explanations for the PAE and the disappearance thereof. The PAE itself can be explained by several hypotheses, e.g., sublethal damage in the bacteria that is due to antibiotic exposure and that needs to be repaired before the normal growth rate returns. Another explanation might be a growth retardation due to free tobramycin which remains inside the bacteria and which is not removed during the washout phase of the PAE experiment. On the other hand, the disappearance of the PAE, which was observed during one interval of dosing with tobramycin (5), may be a result of the outgrowth of resistant subpopulations of bacteria. Alternatively, the phenomenon may be due to

* Corresponding author. Present address: Department of Internal Medicine, Zuiderziekenhuis, Groene Hilledijk 315, 3075 EA Rotterdam, The Netherlands. Phone: 31-10-2903000, ext. 109. Fax: 31-102903361. slow diffusion of tobramycin out of the bacteria or may be a result of a fast repair process in the bacteria. These last processes may happen in the foci of slowly declining antibiotic concentrations from a dosing interval in vivo, while the fast washout of tobramycin during the determination of the PAE in vitro may be too short for these processes to start and have an effect. The PAE may thus be an in vitro phenomenon that does not occur in vivo.

Thus, the most striking difference between the PAE determination in vitro and in vivo is the fast elimination of the antibiotic (i.e., a very short half-life) during in vitro experiments. In order to study the effect of the half-life on the PAE of tobramycin, we determined the PAE during one interval of dosing with tobramycin in an in vitro pharmacokinetic model, simulating tobramycin half-lives in the range of 0.5 to $2.5 \mathrm{~h}$. The PAE was determined at three time points during the interval corresponding to tobramycin concentrations of $20 \times$ the MIC, $5 \times$ the MIC, and $1 \times$ the MIC. Furthermore, we looked for the emergence of resistant subpopulations of bacteria during these PAE determinations, and the hypothesis of the diffusion process was investigated. Finally, to determine whether the larger PAE found in vivo compared to that found in vitro (3) is due to differences in the methods used, the conditions of both methods were simulated in the in vitro model.

(This work was presented at the 37th Interscience Conference on Antimicrobial Agents and Chemotherapy, Toronto, Ontario, Canada, 28 September to 1 October 1997 [6a].)

\section{MATERIALS AND METHODS}

Bacterial strains and media. The strains used were Staphylococcus aureus ATCC 29213 and Pseudomonas aeruginosa ATCC 27853. Mueller-Hinton broth (Difco, Amsterdam, The Netherlands) supplemented with $\mathrm{Ca}^{2+}(25 \mathrm{mg} / \mathrm{liter})$ and $\mathrm{Mg}^{2+}(12.5 \mathrm{mg} /$ liter $)$ was used in all experiments (MHBs). All bacterial samples were plated on Trypticase Soy Agar (TSA; Oxoid, Basingstoke, Hamp- 
shire, England). The MIC of tobramycin was $0.5 \mathrm{mg} /$ liter for both the $S$. aureus strain and the $P$. aeruginosa strain, as determined by a standard macrodilution method with MHBs (19).

Antibiotic. Tobramycin was provided as a solution with a concentration of 10 g/liter (Eli Lilly \& Company, Nieuwegein, The Netherlands). Tobramycin concentrations were assayed by a fluorescence polarization immunoassay with a TDxFLx instrument (Abbott Diagnostic Division, Amstelveen, The Netherlands), which had concentration detection range of 0 to $10 \mathrm{mg} /$ liter and a coefficient of variation of $<6 \%$.

Pharmacokinetic model. The pharmacokinetic model used in this study was previously described in detail (18). Briefly, a two-compartment model consisting of one central compartment and four peripheral compartments including disposable dialyzer units (ST23; Baxter, Utrecht, The Netherlands) was used. The bacteria were exposed in the peripheral compartments to a changing antibiotic concentration that mimicked the pharmacokinetics of tobramycin; the pharmacokinetic profile depended on the half-life chosen for a given experiment.

A volume of $150 \mathrm{ml}$ of a logarithmic-phase culture of $S$. aureus or $P$. aeruginosa (approximately $10^{7} \mathrm{CFU} / \mathrm{ml}$ ) was injected into the peripheral compartments. At time zero a total dose of $15 \mathrm{mg}$ of tobramycin was infused into the central compartment over $1 \mathrm{~h}$ with an infusor (Braun, Melsungen AG, Germany); this resulted in a peak concentration of approximately $10 \mathrm{mg} /$ /iter at $1 \mathrm{~h}$. During the first hour (the infusion time) the half-life was adjusted to $2 \mathrm{~h}$; thereafter, the half-life of tobramycin was set to $0.5,1,1.5,2$, or $2.5 \mathrm{~h}$, as indicated. These different half-lives resulted in a time above the MIC $\left(T_{>\text {MIC }}\right)$ in a range of 3.1 to $11.9 \mathrm{~h}$ and an area under the concentration-time curve (AUC) of 12.3 to $40.2 \mathrm{mg}$. h/liter.

Killing kinetics. To determine the tobramycin concentration and bacterial CFU, samples were taken from each peripheral compartment at time zero, 30 $\min$, and $1 \mathrm{~h}$ and at time points when the tobramycin concentrations were $5 \times$ the MIC and $1 \times$ the MIC. To determine the killing rate, samples were washed two times with cold phosphate-buffered saline (PBS), serially diluted 10-fold in cold PBS, and plated onto TSA plates for colony count determination.

PAE determination. To determine the PAE at different concentrations, samples of $10 \mathrm{ml}$ were taken at the peak concentration $(20 \times$ the MIC) and at the time points corresponding to tobramycin concentrations of $5 \times$ the MIC and $1 \times$ the MIC. An enzymatic method for the inactivation of tobramycin was used (6). Briefly, $10 \mathrm{ml}$ of each sample was added to a freshly prepared solution of tobramycin-acetylating enzyme AAC (3)-II (16) and acetyl coenzyme A, resulting in the inactivation of tobramycin within $5 \mathrm{~min}$. A volume of $5 \mathrm{ml}$ of this solution was immediately placed on ice for one night, to allow free intrabacterial tobramycin to diffuse out of the bacteria, where it would be inactivated by the enzymes. The rate of regrowth in the remaining $5 \mathrm{ml}$ was determined, and the PAE was defined as PAE $=T-C$, where $T$ is the time required for the numbers of CFU in the test culture to increase $1 \log _{10}$ above the count immediately after drug removal and $C$ is the corresponding time for the controls (4). Samples were taken from the PAE regrowth cultures at $0,1,2,3,4$, and $5 \mathrm{~h}$ and serially diluted in cold PBS. Volumes of 0.25 and $0.1 \mathrm{ml}$ were plated onto TSA for the undiluted samples and for the samples with other dilutions, respectively, and the plates were incubated at $37^{\circ} \mathrm{C}$ overnight for $P$. aeruginosa or $48 \mathrm{~h}$ for $S$. aureus. The undiluted samples were also plated onto Iso-Sensitest agar plates (Oxoid), containing tobramycin at concentrations of $0.5,1,2,4$, and $8 \mathrm{mg} /$ liter to detect the presence of resistant subpopulations. Control growth curves were determined in the same in vitro pharmacokinetic model, but without the addition of tobramycin. Control samples were taken at $0,1,2,3,4,5,6,8,10,12,18$, and $24 \mathrm{~h}$ and in the tests with tobramycin at time points when the tobramycin concentrations were $5 \times$ the MIC and $1 \times$ the MIC. The control samples were diluted in MHBs (at $37^{\circ} \mathrm{C}$ ) until the inoculum had the same starting numbers of CFU per milliliter as the test strain at this time. A volume of $10 \mathrm{ml}$ of this control inoculum was treated as described above for the test cultures. All samples that had been placed on ice overnight were quickly brought to $37^{\circ} \mathrm{C}$ as described previously $(7,8)$, and regrowth experiments were performed as described above. All experiments were performed in duplicate on at least two separate occasions.

Simulation of in vivo PAE in mice with renal impairment. To study the differences between the PAE determined in vitro versus that determined in vivo, experimental conditions were simulated as described by Craig et al. (3). Briefly, in their experiments renal impairment was induced in neutropenic mice, resulting in an amikacin half-life of 93.3 to 121 min, thus approaching the pharmacokinetics in humans. For the in vitro simulation of the pharmacokinetics in such mice, the experiments were started as described above, and after $1 \mathrm{~h}$ the half-life was adjusted to $1.5,2.0$, or $2.5 \mathrm{~h}$. During these experiments no enzymatic inactivation of the tobramycin was used, since in the in vivo experiments the PAE is defined as the time that the culture needs to grow $1 \log _{10}$ after the antibiotic concentration declines below the MIC (13). Samples were taken from the peripheral compartments of the in vitro model at $0,1,2,4,6,8,10,12,14,16,24$, and $28 \mathrm{~h}$ and at the time point when the tobramycin concentration was $1 \times$ the MIC. To determine the numbers of CFU per milliliter, the samples were washed twice in cold PBS, diluted serially 10-fold in cold PBS, and plated onto TSA plates. Control growth curves were determined with the model, and at the same time points when the test cultures had a tobramycin concentration of $1 \times$ the MIC, samples were taken and diluted in prewarmed MHBs until the starting inoculum was equal to the starting inoculum of the test culture at that time point, as described above. Regrowth of the control was determined outside the model
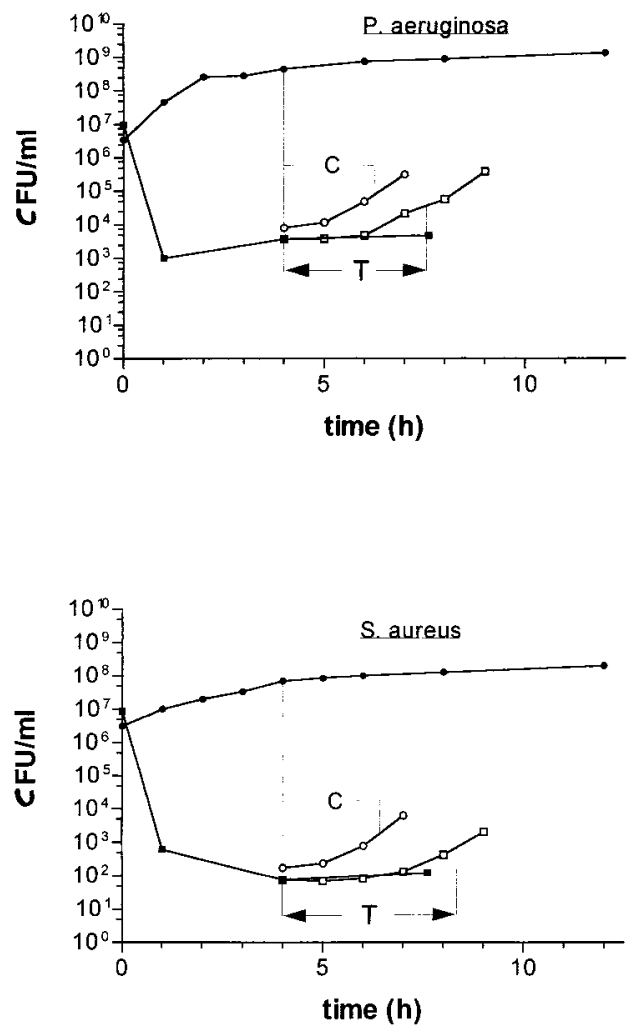

FIG. 1. Representative curves for control growth $(\bullet)$, control regrowth $(\bigcirc)$, killing (ם), and regrowth after killing ( $\square$ ) of $P$. aeruginosa and $S$. aureus determined during all experiments with the pharmacokinetic model. For these curves the samples for PAE measurement were taken when the tobramycin concentration was $5 \times$ the MIC during the simulation with a half-life of $1.5 \mathrm{~h}$. PAE can be determined as PAE $=T-C$, as described by Craig and Gudmundsson (4) (see text for details)

in a test tube containing $10 \mathrm{ml}$. The PAE was determined as described by Gudmundsson et al. (13) by the formula PAE $=T-C-M$, where $M$ represents the time that the concentration in serum exceeds the MIC.

Statistics. The peak concentrations, the half-lives, and the PAE data were analyzed by the Tukey-Kramer multiple comparisons test, one-way analysis of variance, and a test for linear trend between column means with the Instat statistics program (12). A $P$ value of $\leq 0.05$ (two tailed) was considered significant. The correlation between the PAE and other parameters was determined by the parametric correlation test of Pearson.

\section{RESULTS}

Antibiotic concentrations and pharmacokinetics. The observed peak concentration (mean \pm standard error of the mean $[\mathrm{SEM}]$ ) and the concentrations at time points when the tobramycin concentrations were $5 \times$ the MIC and $1 \times$ the MIC were $10.6 \pm 0.3,2.8 \pm 0.1$, and $0.7 \pm 0.1 \mathrm{mg} /$ liter, respectively. The tobramycin half-lives (mean \pm SEM) during the various experiments were $0.6 \pm 0.1,1.1 \pm 0.1,1.7 \pm 0.1,2.2 \pm 0.1$, and $2.6 \pm 0.1 \mathrm{~h}$.

Killing kinetics. Killing and regrowth of $P$. aeruginosa and $S$. aureus were determined in the pharmacokinetic model during all experiments. A representative example of the killing and regrowth curves for both strains exposed to tobramycin with a half-life of $1.5 \mathrm{~h}$ is shown in Fig. 1. Figure 1 also shows examples of a control growth curve and the control regrowth curve. If the control regrowth curve is compared with the control growth curve in the model, it seems to have a short lag phase before regrowth begins exponentially. 

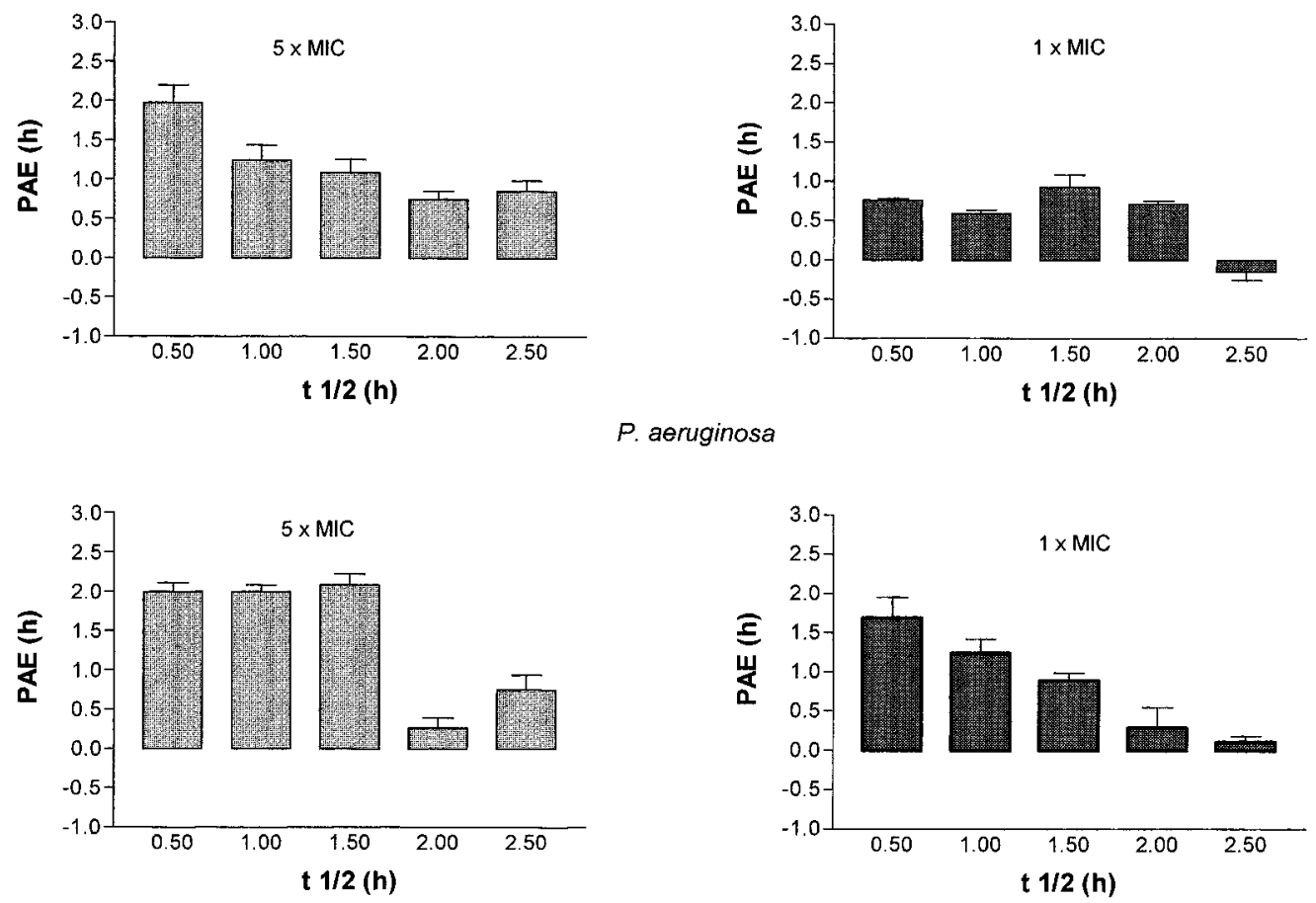

S. aureus

FIG. 2. Mean \pm SEM PAEs determined with concentrations of $5 \times$ the MIC and $1 \times$ the MIC for $P$. aeruginos $a$ and for $S$. aureus, respectively, during the simulation with different half-lives $\left(t_{1 / 2}\right)$ of tobramycin.

PAE determination. A summary of all mean \pm SEM PAEs determined in the various experiments is given in Fig. 2. The mean \pm SEM PAEs for $P$. aeruginosa and $S$. aureus determined at the peak concentration $(20 \times$ the MIC) were $2.0 \pm 0.7$ and $1.8 \pm 0.5 \mathrm{~h}$, respectively, which are comparable to those published previously (5), and were not significantly different between the different experiments. The PAEs for $P$. aeruginosa at a tobramycin concentration of $5 \times$ the MIC depended on the tobramycin half-life chosen. A significantly decreasing linear trend $(P<0.0001)$ was found, from a mean \pm SEM PAE of $2.0 \pm 0.2 \mathrm{~h}$ to one of $0.8 \pm 0.3 \mathrm{~h}$ for half-lives of 0.5 and $2.5 \mathrm{~h}$, respectively. At a concentration of $1 \times$ the MIC, the PAE was about $0.7 \mathrm{~h}$ for half-lives of 0.5 to $2.0 \mathrm{~h}$ but completely disappeared if the tobramycin half-life was set at $2.5 \mathrm{~h}$ (Fig. 2). In experiments with $S$. aureus and a tobramycin concentration of $5 \times$ the MIC, the PAEs showed no significant difference for half-lives of $0.5,1$, and $1.5 \mathrm{~h}$, but a significant decrease in the PAEs was observed for half-lives of 2 and $2.5 \mathrm{~h}(P<0.001)$. At a concentration of $1 \times$ the MIC the PAEs for $S$. aureus decreased from $1.7 \pm 0.5$ to $0.1 \pm 0.2 \mathrm{~h}$, resulting in a significant linear trend $(P<0.0001)$. The data presented in Fig. 2 also indicate that the PAEs for both $P$. aeruginosa and $S$. aureus decline during a dosing interval. For $P$. aeruginosa the PAE was a significant linear declining trend $(0.0001<P \leq 0.01)$ during the dosing interval for half-lives of 1.0 to $2.5 \mathrm{~h}$. For $S$. aureus the PAE also decreases during the dosing interval, but a significant linear decrease in PAE is observed only for half-lives of 2.0 and $2.5 \mathrm{~h}(P=0.004$ and $P<0.0001$, respectively).

The linear declining trend seen with an increase in the halflife is also shown by the good correlations between the AUC and the PAE. The AUC determined for the time period until the concentration declines below the MIC correlates well with the PAE at $1 \times$ the MIC for $S$. aureus $(r=-0.99 ; P<0.001)$ and with the PAE at $5 \times$ the MIC for $P$. aeruginosa $(r=0.90$; $P=0.039$ ).

PAE determination after incubation on ice. To study the effect of free intrabacterial tobramycin, the PAE was determined after an overnight incubation on ice, and the $\triangle \mathrm{PAE}$ $(\triangle \mathrm{PAE}=$ direct $\mathrm{PAE}-\mathrm{PAE}$ after incubation on ice $)$ was calculated for $P$. aeruginosa and $S$. aureus. The overall $\triangle \mathrm{PAEs}$ (mean \pm SEM) were $0.3 \pm 0.5 \mathrm{~h}(P<0.0001)$ and $0.3 \pm 0.4 \mathrm{~h}$ $(P<0.0001)$ for $P$. aeruginosa and $S$. aureus, respectively, indicating a slight but significantly lower PAE when the PAE was determined after incubation on ice. Differentiation of the $\triangle$ PAEs determined when the concentration was at its peak versus those determined when the concentrations were at $5 \times$ the MIC and $1 \times$ the MIC indicated that the effect of additional incubation on ice was mainly observed for the PAEs calculated when the concentration was $5 \times$ the MIC (mean \pm SEM $\Delta \mathrm{PAE}=0.5 \pm 0.5 \mathrm{~h}$ for both $P$. aeruginosa and $S$. aureus).

Regrowth of resistant subpopulations. No resistant subpopulations of $P$. aeruginosa were found during any of the experiments. For $S$. aureus all samples showed growth on the plates containing 0.5 and $1.0 \mathrm{mg}$ of tobramycin per liter. However, the samples used to generate control growth curves and plated on the same plates also showed the same growth on these plates. This would indicate that there may be a subpopulation in the starting inoculum for which the MIC is slightly higher. Another phenomenon, seen for $S$. aureus only, was the growth of small colony variants. These colony types were visible only after incubation for $48 \mathrm{~h}$ at $37^{\circ} \mathrm{C}$. The MIC of tobramycin for these small colonies by an E-test (AB-Biodisk, Solna, Sweden) reached 2 to $4 \mathrm{mg} /$ liter. The generation time in MHBs at $37^{\circ} \mathrm{C}$ for the small colony variants showed a range of 55.0 to $60.3 \mathrm{~min}(n=4)$ compared to a generation time of $35 \mathrm{~min}$ for the original test strain and for the large colony types after 


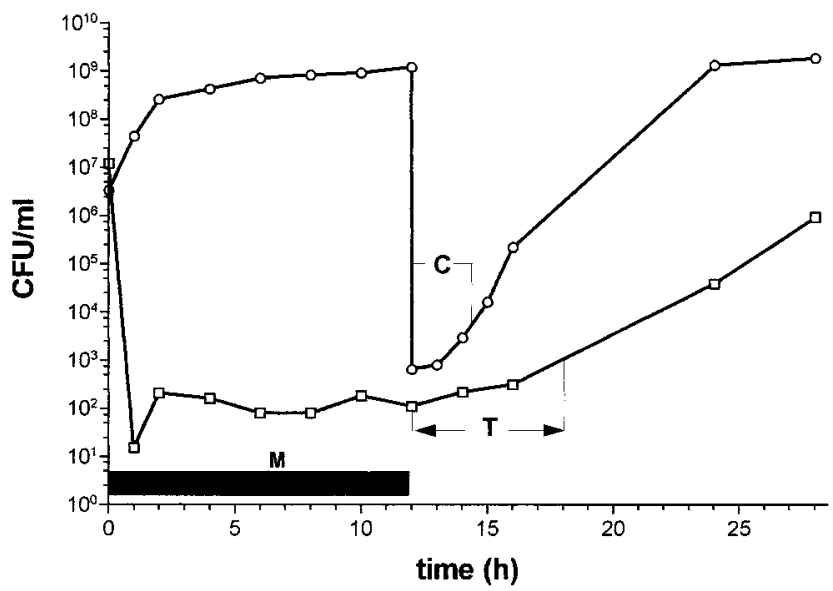

FIG. 3. Representative control growth $(\bigcirc)$ and killing $(\square)$ curves for P. aeruginosa in the in vitro model during a simulation of the pharmacokinetics of tobramycin in mice with renal impairment determined with a half-life of $2.5 \mathrm{~h}$. PAE was determined as PAE $=T-C-\mathrm{M}$ (13), where $T$ is the time for the drug exposed bacteria to grow $1 \log _{10} \mathrm{CFU}, C$ is the corresponding time for the nonexposed control, and $M$ is the time above the MIC.

treatment $(n=4)$. The DNAs of both the large and the small colony types were typed by random amplification of polymorphic DNA (RAPD) by previously described protocols (23), using RAPD 1, RAPD 7, and ERIC II as primers, to check whether the small and large colony variants were genetically identical. Since no variability in the amplification patterns was observed, genetic differences between the two variants were either small or absent.

Simulation of in vivo PAE in mice with renal impairment. A representative example of a killing curve for $P$. aeruginosa, combined with its growth curve, as determined in the pharmacokinetic model after one tobramycin dosing interval, is shown in Fig. 3. These curves indicate the existence of a prolonged regrowth time after the concentration has declined below the MIC, resulting in long PAEs. The mean PAEs for the strains determined during those simulation experiments are presented in Table 1. These data were compared with the PAE values determined when the concentration was at its peak $(20 \times$ the MIC) and at $1 \times$ the MIC, as determined during the experiments described above. This shows a long PAE for Pseudomonas, when simulating the in vivo circumstances, which is even longer than the PAE determined at $20 \times$ the MIC. For both strains the PAEs determined during the simulation of the in vivo situation were longer than those determined with $1 \times$ the MIC.

\section{DISCUSSION}

In a previous article we described the fact that the PAEs for $P$. aeruginosa and $S$. aureus disappeared during one interval of tobramycin dosing (5). However, the mechanism behind this phenomenon was not clear. The phenomenon of the PAE can be explained by several mechanisms. One is that sublethal damage in the exposed but surviving bacteria needs to be repaired before regrowth can start. It could also be that some free tobramycin remains inside the bacteria and causes growth retardation. The first explanation has been investigated by others $(1,9,10)$, and their results indicate that DNA, RNA, and protein syntheses are all inhibited during exposure to tobramycin. For Pseudomonas DNA and RNA syntheses recover rapidly during the PAE phase and a large increase in DNA synthesis is seen shortly before logarithmic regrowth starts. In contrast, protein synthesis was inhibited throughout the PAE phase and was resumed only after the bacteria were again in the logarithmic phase of growth. The conclusions that can be drawn from these observations could be that sublethal damage is induced by tobramycin exposure, that the damage needs time to be repaired, and that this time correlates with the PAE. In an earlier observation, Gottfredsson et al. (11) observed that tobramycin induced ultrastructural changes, such as a pattern of dense nuclear material and peripheral vacuoles, and that these changed during the PAE phase and disappeared at the end of the PAE phase. This also indicates that during the PAE phase repair mechanisms are working in order to restore the normal growth rate.

The second possibility was partly investigated in this study by determining the PAE after overnight incubation on ice, thus allowing the free tobramycin inside the bacteria to diffuse out, where it would be eliminated by the tobramycin-acetylating enzymes (these enzymes also work at $0^{\circ} \mathrm{C}$; data not shown). Our results indicate that free intrabacterial tobramycin has only a very minor effect. The largest effect of intrabacterial tobramycin was seen in the experimental groups with the longest exposure time at a relatively high tobramycin concentration (samples taken when the concentration was $5 \times$ the MIC). The PAE thus seems to be predominantly due to sublethal damage, reflected by inhibition of DNA, RNA, and protein syntheses during the PAE phase, rather than due to free intrabacterial tobramycin.

During the PAE determinations the control regrowth curves seem to have a little lag phase, compared to the control growth curve in the model, before exponential regrowth begins. This may be explained by an inoculum effect due to dilution of the control sample inoculum to the starting inoculum of the test strains. Furthermore, at the time that the control samples were taken the growth of the control was at the start of a stationary phase, as indicated by the flattening of the control growth curve. Another explanation for the growth rate difference may be that the control growth was determined in the in vitro model, in which the growth rate is slightly faster (unpublished data), while the regrowth of the control was determined in a tube.

The repair processes which take place during the PAE period may well start at the time that the tobramycin concentrations are declining toward the MIC. The PAE in vitro is normally determined after a short on-off exposure to the antibiotic. This fast removal corresponds to a very short half-life of the antibiotic, which may be too short for a repair process to have started. By varying the half-life of tobramycin over a range of 0.5 to $2.5 \mathrm{~h}$, the time available for this repair process

TABLE 1. Comparison of tobramycin PAEs for $P$. aeruginosa and $S$. aureus determined in vitro and during simulation of in vivo PAE

\begin{tabular}{lccrc}
\hline \multirow{2}{*}{ Strain } & $\begin{array}{c}\text { Half-life } \\
(\mathrm{h})\end{array}$ & $20 \times$ the MIC & $1 \times$ the MIC & $\begin{array}{c}\text { Simulation of } \\
\text { in vivo PAE }\end{array}$ \\
\cline { 3 - 5 } & & & & \multicolumn{3}{c}{ PAE $(\mathrm{h})^{a}$} \\
\hline P. aeruginosa & 1.5 & $2.0 \pm 0.8$ & $0.9 \pm 0.3$ & $6.1 \pm 0.5$ \\
& 2.0 & $1.7 \pm 0.3$ & $0.7 \pm 0.1$ & $5.1 \pm 0.1$ \\
& 2.5 & $2.5 \pm 1.0$ & $-0.2 \pm 0.3$ & $5.0 \pm 1.5$ \\
& & & & \\
S. aureus & 1.5 & $1.7 \pm 0.1$ & $0.9 \pm 0.2$ & $2.3 \pm 1.0$ \\
& 2.0 & $1.3 \pm 0.3$ & $0.3 \pm 0.5$ & $4.0 \pm 1.3$ \\
& 2.5 & $2.2 \pm 0.8$ & $0.1 \pm 0.2$ & $1.3 \pm 0.1$ \\
\hline
\end{tabular}

${ }^{a}$ Data are means \pm SEMs for two separate experiments. 
to start can be influenced. In the present study, the overall effect of the half-life of tobramycin on the PAE is that the PAE diminishes with increasing half-lives and finally disappears. This indicates that for short half-lives, there is insufficient time for this repair process to have an effect, so a PAE is still present at the end of the dosing interval, while for longer half-lives this time for repair is prolonged, resulting in the disappearance of the PAE for a tobramycin half-life of 2 to $2.5 \mathrm{~h}$ or longer. The minimum half-life needed for the PAE is clearly strain dependent, as would be expected. Stronger evidence for the existence of repair mechanisms, which are active during therapy and before the concentration declines below the MIC, would be the observation of ultrastructural changes, as mentioned earlier. For a further confirmation of our hypothesis concerning the repair mechanism, our experiments need to be combined with those of Gottfredson and colleagues (9-11).

Shorter half-lives of tobramycin result in smaller AUCs and a shorter $T_{>\text {MIC }}$. The correlation seen between the increasing half-life and the decline in the PAE can thus also be expressed as a correlation between the AUC and PAE or the $T_{>\text {MIC }}$ and PAE. Since only one dose was administered in these experiments, it is not possible to determine the most important parameter for the disappearance of the PAE. Extrapolation of the PAE data and the different AUCs by linear regression analysis showed that the AUC had to be $40.4 \mathrm{mg} \cdot \mathrm{h} /$ liter for the PAE to become zero for $S$. aureus. However, linear regression showed a reasonable fit only for $S$. aureus $(r=0.86)$, so with these data no unifying AUC could be determined to explain the disappearance of the PAE during therapy. The same was true for $T_{>\mathrm{MIC}}$ and PAE.

The influence of the half-life on the disappearance of the PAE seems contradictory for the PAE determined in vivo. Craig et al. (3) showed in a mouse model that the PAE of amikacin for $P$. aeruginosa was prolonged when they used a half-life that was increased to 1.5 or $2.0 \mathrm{~h}$ instead of the normal half-life of 18 to $32 \mathrm{~min}$. In animal studies the PAE, by definition, is determined as delayed bacterial regrowth compared to the growth of a control after the concentration of the antibiotic falls below the MIC. This is precisely the difference between the methods for PAE determination in vivo and in vitro. In the in vitro PAE test the delay in regrowth is measured in antibiotic-free medium, while in vivo this delayed regrowth is determined at sub-MICs. This may partly explain why the PAE determined in vivo in mice with normal renal clearance is longer than the PAEs measured in vitro, since this may be a combination of a PAE and a postantibiotic sub-MIC effect (PA-SME). Increasing the half-life in mice by inducing renal impairment results in an even longer in vivo PAE (3). The results presented above (i.e., longer PAEs in mice with renal impairment) may thus be explained by the longer exposure of microorganisms to sub-MICs and would better be described as such. By simulation of both in vivo and in vitro PAE determination methods in our in vitro model, the roles of these subMICs could be studied. With increasing half-lives in the in vitro model, the PAE disappeared, while during simulation of the in vivo PAE determination method, the PAE was still present and was even longer than the PAE determined under standard in vitro circumstances. The in vivo PAE thus correlates with the PA-SME, as described by Odenholt-Tornqvist et al. (21). Furthermore, that group of investigators recently showed (17) that there is a significant difference between PAE and PA-SME for Streptococcus pyogenes and penicillin when pharmacokinetics in humans are simulated in an in vitro model.

Although the PAE values simulated in vivo approach the values seen in animal models, they are not completely equal, indicating that other environmental circumstances in vivo, such as different generation times, may also be responsible for the in vivo PAE. This should be investigated with different enrichments of the medium used in vitro that would produce a generation that approaches the in vivo generation time. Until this has been investigated, the importance of these environmental circumstances is not clear, but the sub-MICs during the determination of the in vivo PAE seem to be of major importance.

Another explanation for the previous findings might be the regrowth of resistant subpopulations. In the present study no resistant subpopulations (or small colony variants) were found for $P$. aeruginosa. This may be explained by the fact that the agar plates containing $P$. aeruginosa samples were incubated overnight and not for $48 \mathrm{~h}$, as was done for $S$. aureus. This was done because the $P$. aeruginosa colonies grew too fast for them to be incubated longer than $24 \mathrm{~h}$. For $S$. aureus small colony variants were isolated, and for these isolates the MIC was slightly increased, but the isolates were not resistant to tobramycin. This appearance of a less susceptible subpopulation is thus not likely to explain the disappearance of the PAE during the dosing interval, since these small colony variants show an increased generation time compared to that for the test strain; the regrowth of the small colony types would thus result in a prolongation rather than a disappearance of the PAE. There was no correlation between the appearance of the small colony variants and any of the variables during the experiments, such as the half-life, AUC, $T_{>\text {MIC }}$, sample time, or PAE value. The fact that we did not find small colony variants for $P$. aeruginosa is due to technical circumstances and is not in contradiction with earlier findings (8).

The observations presented above indicate that the PAE of tobramycin determined in vitro under standard conditions has no clinical relevance, since its supposed effect vanishes at the end of the dosing interval, just as it is supposed to start being important. The PAEs of antibiotics exceeding the dosing interval could still be of relevance. The PAE determined in vivo is possibly, for the major part, a description of a sub-MIC effect that is further influenced by environmental circumstances in vivo. This in itself is clinically relevant, since it describes the regrowth of bacteria during therapy. The PA-SME thus represents only the regrowth inhibition found when the antibiotic concentration falls below the MIC.

\section{ACKNOWLEDGMENTS}

We thank A. van Belkum and E. A. H. Panken for help in typing the DNAs of the different colony variants of $S$. aureus.

\section{REFERENCES}

1. Barmada, S., S. Kohlepp, J. Leggett, R. Dworkin, and D. Gilbert. 1993. Correlation of tobramycin-induced inhibition of protein synthesis with postantibiotic effect in Escherichia coli. Antimicrob. Agents Chemother. 37:26782683.

2. Craig, W. A. 1993. Post-antibiotic effects in experimental infection models: relationship to in-vitro phenomena and to treatment of infections in man. J. Antimicrob. Chemother. 31(Suppl. D):149-158.

3. Craig, W. A., J. Redington, and S. C. Ebert. 1991. Pharmacodynamics of amikacin in vitro and in mouse thigh and lung infections. J. Antimicrob. Chemother. 27(Suppl. C):29-40.

4. Craig, W. A., and S. Gudmundsson. 1986. The postantibiotic effect, p. 515 536. In V. Lorian (ed.), Antibiotics in laboratory medicine, 2nd ed. The Williams \& Wilkins Co., Baltimore, Md.

5. den Hollander, J. G., J. W. Mouton, M. P. J. van Goor, F. P. Vleggaar, and H. A. Verbrugh. 1996. Alteration of the postantibiotic effect during one dosing interval of tobramycin, simulated in an in vitro pharmacokinetic model. Antimicrob. Agents Chemother. 40:784-786.

6. den Hollander, J. G., J. W. Mouton, I. A. J. M. Bakker-Woudenberg, F. P. Vleggaar, M. P. J. van Goor, and H. A. Verbrugh. 1996. An enzymatic method for inactivation of aminoglycosides during postantibiotic effect measurement. Antimicrob. Agents Chemother. 40:488-490. 
6a.den Hollander, J. G., K. Fuursted, H. A. Verbrugh, and J. W. Mouton. 1997. The clinical relevance of the postantibiotic effect of aminoglycosides is questionable, abstr. A-84a, p. 17. In Program and abstracts of the 37th Interscience Conference on Antimicrobial Agents and Chemotherapy. American Society for Microbiology, Washington, D.C.

7. Eagle, H., and A. D. Musselman. 1949. The slow recovery of bacteria from the toxic effects of penicillin. J. Bacteriol. 58:475-490.

8. Gerber, A. U., and W. A. Craig. 1981. Growth kinetics of respiratory pathogens after short exposures to ampicillin and erythromycin in vitro. J. Antimicrob. Chemother. 8(Suppl. C):81-91.

9. Gottfredsson, M., H. Erlendsdóttir, A. Gudmundsson, and S. Gudmundsson. 1996. Determination of in vitro effects in Staphylococcus aureus and Escherichia coli by $\left[{ }^{3} \mathrm{H}\right]$ thymidine incorporation. Clin. Microbiol. Infect. 2: 99-104.

10. Gottfredsson, M., H. Erlendsdóttir, A. Gudmundsson, and S. Gudmundsson. 1995. Different patterns of bacterial DNA synthesis during postantibiotic effect. Antimicrob. Agents Chemother. 39:1314-1319.

11. Gottfredsson, M., H. Erlendsdóttir, R. Kolka, A. Gudmundsson, and S. Gudmundsson. 1993. Ultrastructural alterations of bacteria during the postantibiotic effect. Chemotherapy (Basel) 39:153-162.

12. Graphpad Instat. 1990. Instat 2 program manual. Graphpad Software Inc. San Diego, Calif.

13. Gudmundsson, S., S. Einarsson, H. Erlendsdottir, J. Moffat, W. Bayer, and W. A. Craig. 1993. The post-antibiotic effect of antimicrobial combinations in a neutropenic murine thigh infection model. J. Antimicrob. Chemother. 31(Suppl. D):177-191.

14. Isaksson, B., L. Nilsson, R. Maller, and L. Soren. 1988. Postantibiotic effect of aminoglycosides on gram-negative bacteria evaluated by a new method. J. Antimicrob. Chemother. 22:23-33.

15. Kapusnik, J. E., C. J. Hackbarth, H. F. Chambers, T. Carpenter, and M. A. Sande. 1988. Single, large, daily dosing versus intermittent dosing of tobramycin for treating experimental Pseudomonas pneumonia. J. Infect. Dis. 158: $7-12$.
16. Leitner, F., and K. E. Price. 1982. Aminoglycosides under development, p 29-36. In A. Whelton and H. C. Neu (ed.), The aminoglycosides, 1st ed. Marcel Dekker Inc., New York, N.Y.

17. Löwdin, E., I. Odenholt, S. Bengtsson, and O. Cars. 1996. Pharmacodynamic effects of sub-MICs of benzylpenicillin against Streptococcus pyogenes in a newly developed in vitro kinetic model. Antimicrob. Agents Chemother. 40: 2478-2482.

18. Mouton, J. W., and J. G. den Hollander. 1994. Killing of Pseudomonas aeruginosa during continuous and intermittent infusion of ceftazidime in an in vitro pharmacokinetic model. Antimicrob. Agents Chemother. 38:931936.

19. National Committee for Clinical Laboratory Standards. 1990. Methods for dilution antimicrobial susceptibility tests for bacteria that grow aerobically, 2nd ed. Approved standard M7-A2. National Committee for Clinical Laboratory Standards, Villanova, $\mathrm{Pa}$.

20. Nicolau, D. P., C. D. Freeman, P. B. Belliveau, C. H. Nightingale, J. W. Ross, and R. Quintiliani. 1995. Experience with a once-daily aminoglycoside program administered to 2,184 adult patients. Antimicrob. Agents Chemother. 39:650-655.

21. Odenholt-Tornqvist, I., E. Lödwin, and O. Cars. 1992. The post-antibiotic sub-MIC effect of vancomycin, roxithromycin, sparfloxacin, and amikacin. Antimicrob. Agents Chemother. 36:1852-1858.

22. Ter Braak, E. W., P. J. de Vries, K. P. Bouter, S. G. van der Vegt, G. C Dorrestein, J. W. Nortier, A. van Dijh, R. P. Verkooyen, and H. A. Verbrugh. 1990. Once-daily dosing regimen for aminoglycoside plus beta-lactam combination therapy of serious bacterial infections: a comparative trial with netilmicin plus ceftriaxone. Am. J. Med. 89:58-66.

23. Van Belkum, A., J. Kluytmans, W. van Leeuwen, R. Bax, W. Quint, E. Peters, A. Fluit, C. VandenBroucke-Grauls, A. van den Brule, H. Koeleman, W. Melchers, J. Meis, A. Elaichouni, M. Vaneechoutte, F. Moonens, N. Maes, M. Struelens, F. Tenover, and H. Verbrugh. 1995. Multicenter evaluation of arbitrarily primed PCR for typing of Staphylococcus aureus strains. J. Clin. Microbiol. 33:1537-1547. 